\title{
Caracterização fenotípica pelo antibiograma de cepas de Escherichia coli isoladas de manipuladores, de leite cru e de queijo “Minas Frescal” em um laticínio de Goiás, Brasil
}

\author{
Phenotypic characterization by antibiogram of Escherichia coli strains isolated from handlers, raw \\ milk and "Minas Frescal" cheese samples in a dairy process \\ plant in Goiás, Brazil
}

\author{
Maria Raquel Hidalgo Campos ${ }^{*}$ André Kipnis² Maria Cláudia Dantas Porfírio Borges Andrée $^{2}$ \\ Carla Atavila da Silva Vieira ${ }^{2}$ Liana Borges Jayme ${ }^{1}$ \\ Patrícia Pimentel Santos ${ }^{2}$ Álvaro Bisol Serafini ${ }^{2}$
}

RESUMO

Este trabalho objetivou caracterizar fenotipicamente isolados de Escherichia coli a partir de amostras obtidas de mãos e narizes de manipuladores, de leite cru e de queijo Minas Frescal produzidos em um laticínio em Goiás, Brasil, visando a estabelecer uma relação de contaminação entre o manipulador e/ou a matéria-prima e o produto final. Foram coletadas 24 amostras de leite cru e de queijo Minas Frescal, 46 swabs de mãos e 46 de narizes dos manipuladores envolvidos na fabricação do queijo, no período entre março/2004 e fevereiro/2005. As 69 cepas isoladas foram submetidas ao teste de suscetibilidade a seis antimicrobianos (ampicilina, cefalotina, ciprofloxacina, gentamicina, sulfametoxazol trimetoprin e tetraciclina) e, a partir deste teste, foi possível agrupá-las em 17 diferentes perfis fenotípicos (A$Q$ ). Somente um perfil fenotípico (E) foi semelhante entre cepas de $\boldsymbol{E}$. coli isoladas, tanto de amostra de queijo $\left(14 Q^{b}\right)$ como de um dos manipuladores $\left(10 \mathrm{M}^{1}\right)$. Tal resultado sugere uma possível contaminação do produto durante a sua manipulação; portanto, apenas neste caso pôde-se estabelecer a origem da E. coli para o queijo. Nas demais situações, não foi possível determinar a fonte da bactéria para o queijo. Conclui-se que o antibiograma não se mostrou um teste eficiente na determinação da fonte de microrganismos para o produto final.

Palavras-chave: Coliformes, manipulação, leite cru, queijo Minas Frescal.

\section{ABSTRACT}

The present work aimed to type $\boldsymbol{E}$. coli strains obtained from handlers hands and noses, raw milk and Minas Frescal cheese produced by a dairy milk processing plant in Goiás, Brazil in order to establish a contamination relationship between the sources of $\mathbf{E}$. coli and cheese. Twenty-four samples were collected from raw milk and Minas Frescal cheese, 46 from hands and 46 from noses of handlers involved in the cheese manufacturing, between March/2004 and February/ 2005. All 69 E. coli strains isolated were submitted to the susceptibility test for six antimicrobials (ampicillin, cephalothin, ciprofloxacin, gentamicin, trimethopim-sulphamethoxazole and tetracycline. The strains were grouped in 17 different phenotypic susceptibility profiles (A-Q). The test allowed to establish correlation among a cheese strain $\left(14 Q^{b}\right)$ and a handler $\left(10 M^{1}\right)$ strain that presented the same susceptibility profile (E) suggesting a possible product contamination during the handling. In conclusion, the phenotypic susceptibility profiles determinated by the antibiogram method failed to establish a correlation between the strains obtained from the probable sources (handlers and milk) and the final products.

Key words : Coliforms, handling, raw milk, Minas Frescal cheese.

\section{INTRODUÇÃO}

O leite é considerado, devido à sua composição química, um excelente meio de cultura, podendo ser facilmente contaminado por vários microrganismos (DOYLE et al., 1997; CHYE et al., 2004). Portanto, o leite deve ser obtido com a máxima higiene e mantido em baixa temperatura, desde a ordenha até o seu beneficiamento, visando a garantir as características físicas, químicas e nutricionais (BONFOH et al., 2003). A composição do leite, sua microbiota natural, a contaminação pós-pasteurização, o processamento e manipulação, os equipamentos, a temperatura inadequada durante estocagem e o transporte podem resultar em altos níveis de

${ }^{1}$ Faculdade de Nutrição, Universidade Federal de Goiás (UFG), Goiânia, Goiás, Brasil. Rua 23, n. 243, 704, Setor Central, 74015120, Goiânia, Goiás, Brasil. E-mail: raq7@brturbo.com.br. *Autor para correspondência.

${ }^{2}$ Instituto de Patologia Tropical e Saúde Pública,UFG, Goiânia, Goiás, Brasil. 
microrganismos patogênicos em queijos (ARAÚJO et al., 2002).

No Brasil, a indústria de laticínios é expressiva. Em 2003, foram produzidos 22.254 milhões de litros de leite (IBGE, 2003) e em 2002, foram produzidas 31.762 toneladas de queijo Minas Frescal (BARROS et al, 2004).

O queijo Minas Frescal é um queijo branco, semelhante ao "Queso Blanco" fabricado em outros países da América Latina, produzido a partir de leite de vaca pasteurizado, caracterizado por alta atividade água, baixo pH $(5,1-5,6)$ e 1 a 6\% de Cloreto de Sódio (NaCl) (FREITAS et al., 1993). Possui cerca de 43\% a $55 \%$ de umidade e uma vida de prateleira de 10 a 14 dias (GONZALEZ et al., 2000). Na sua fabricação, utiliza-se coalho enzimático ou químico, remove-se o soro e realiza-se a moldagem e a salga (ARAÚJO et al., 2002). Esse queijo tem ampla aceitação comercial e faz parte do hábito alimentar da população das diversas regiões do país (LOGUERCIO \& ALEIXO, 2001; ALMEIDA FILHO et al., 2002; CÂMARA et al., 2002; CARDOSO \&ARAÚJO, 2004; ROOS et al., 2005). Destaca-se que geralmente é fabricado a partir de leite cru, sendo um problema em saúde pública, pois constitui-se em veículo para inúmeros agentes etiológicos de enfermidades zoonóticas, entre eles a $\boldsymbol{E}$. coli (ARAÚJO et al., 2002; SCHOUTEN et al., 2004).

Segundo DE BUYSER et al. (2001), é difícil estimar a proporção de doenças transmitidas por leite e derivados, devido às limitações dos sistemas de vigilância. Na França, o leite e os seus derivados estiveram envolvidos em 5\% dos 3.839 surtos de doenças transmitidas por alimentos de origem bacteriana entre 1988 e 1997 . Neste país, dos 60 surtos relatados, $48 \%$ foram relacionados ao leite cru. Do mesmo modo, de 1 a $5 \%$ dos casos relatados em outros países como Escócia, Inglaterra e País de Gales (COWDEN et al., 1995; AMMON, 1997), Estados Unidos (BEAN et al., 1997) e Holanda (SIMONE et al., 1997) estiveram relacionados ao leite cru.

No Brasil, tem-se evidenciado a presença de microrganismos patogênicos em queijo Minas Frescal, sendo amplamente reconhecida a presença de coliformes fecais neste produto em vários estudos realizados em diferentes locais, como em Belo Horizonte/ MG (PEREIRA et al., 1999), em Cuiabá/MT (LOGUERCIO \& ALEIXO, 2001), em Poços de Caldas/ MG (ALMEIDA FILHO \& NADER FILHO, 2002), em cidades do interior do Paraná (KOTTWITZ \& GUIMARÃES, 2003), no Rio de Janeiro/RJ (BARROS et al., 2004), no Distrito Federal (CARDOSO \& ARAÚJO, 2004) e em Três Passos/RS (ROOS et al., 2005).
A $\boldsymbol{E}$. coli faz parte da microbiota entérica de mamíferos e aves, sendo uma bactéria Gram-negativa pertencente à família Enterobacteriaceae (BRENER, 1984). É isolada com freqüência em alimentos e em produtos lácteos, incluindo os armazenados sob refrigeração (NATARO \& KAPER, 1998; FDA, 2002).

Nas últimas duas décadas, muitos métodos têm sido aplicados para comparar cepas de $\boldsymbol{E}$. coli na tentativa de identificar os mecanismos de transmissão e as fontes de contaminação (ZADOKS et al., 2002). Entre as técnicas fenotípicas utilizadas, o teste de suscetibilidade a antimicrobianos tem sido especialmente usado devido a seu baixo custo e à facilidade de execução, além de contribuir para informar sobre a resistência microbiana (KLUYTMANS et al., 1995; ACCO et al., 2003).

O objetivo deste estudo foi caracterizar fenotipicamente, por meio do teste de suscetibilidade in vitro, as cepas de $\boldsymbol{E}$. coli isoladas das amostras de manipuladores de leite cru e de queijo Minas Frescal analisadas e determinar a relação de contaminação, se presente, entre a matéria-prima (leite cru), ou o manipulador e o produto final (queijo).

\section{MATERIAL E MÉTODOS}

No período de março de 2004 a fevereiro de 2005, foram coletadas 140 amostras em um laticínio no estado de Goiás, sendo: 24 amostras de leite cru (não pasteurizado), 24 amostras de queijo ( $\pm 500 \mathrm{~g}$ ), 46 amostras de mãos e 46 de nasofaringe obtidos de quatro manipuladores, funcionários da área de processamento de queijo. As amostras foram colhidas quinzenalmente (uma de leite cru, uma de queijo fabricado a partir do mesmo leite coletado, uma da nasofaringe e uma das mãos de cada manipulador que trabalhava no dia da coleta), segundo os critérios estabelecidos por MIDURA\& BRYANT(2001).

As análises microbiológicas foram realizadas no Laboratório de Microbiologia de Alimentos da Universidade Federal de Goiás e se basearam nas técnicas descritas no FDA (2002). Foram feitas as seguintes determinações: análise presuntiva para coliformes, coliformes fecais e $\boldsymbol{E}$. coli; análise confirmativa para coliformes; completa para $\boldsymbol{E}$. coli (coloração de Gram e provas do IMViC). Como teste fenotípico de tipagem bacteriana, foi realizado o teste de suscetibilidade a antimicrobianos de todos isolados, usando a técnica de difusão em placas segundo NCCLS (2003). Os antibióticos usados foram ampicilina, cefalotina, ciprofloxacina, gentamicina, sulfametoxazol trimetoprin e tetraciclina. A interpretação dos resultados foi feita de acordo com os padrões do NCCLS (2003). 


\section{RESULTADOS E DISCUSSÃO}

Na tabela 1, pode ser observado que um total de 69 cepas de $\boldsymbol{E}$. coli foram isoladas das 47 amostras positivas de leite cru, de queijo Minas Frescal, de nasofaringe e das mãos de manipuladores do laticínio local do estudo. A presença de diversas cepas de $\boldsymbol{E}$. coli indica que essa contaminação pode ser de origem fecal e, portanto, esse alimento está em condições higiênicas insatisfatórias, representando risco direto à saúde humana e animal (NATARO \& KAPER, 1998; BABÁK et al., 2005).

Contaminação

Manipuladores - O laticínio estudado contava, à época da pesquisa, com quatro funcionários que trabalhavam diretamente na fabricação do queijo, codificados em algarismos 1, 2, 3 e 4 . Dos quatro manipuladores, três (75\%) apresentaram-se contaminados, pelo menos uma vez, nas mãos e/ou no nariz durante o período da coleta. Das 46 amostras obtidas da nasofaringe e das 46 obtidas a partir das mãos dos manipuladores investigados, foram isoladas 11 (12\%) cepas de $\boldsymbol{E}$. coli, sendo três $(6,5 \%)$ da nasofaringe e oito $(17,4 \%)$ das mãos (Tabela 1$)$.

Esses resultados mostram porcentagens de contaminação superiores aos obtidos em outras pesquisas. A Organização Mundial de Saúde relata que $60 \%$ das doenças de origem alimentar são provocadas por microrganismos, sendo o manipulador o principal veículo dessa transmissão (SILVAJR., 2002). CURTIS et al. (2000) encontraram $\boldsymbol{E}$. coli nas mãos de $21,9 \%$ dos manipuladores de um restaurante em Caracas, Venezuela. Já no estudo realizado por CARDOSO (1999), foi encontrada uma porcentagem superior (97,3\%) de $\boldsymbol{E}$. coli em mãos de manipuladores de restaurantes da Bahia.

A E c coli faz parte da microbiota do trato gastrointestinal humano desde o nascimento. A partir daí, pode disseminar para outras regiões do corpo e conseqüentemente para os alimentos, quando produzidos sem os conhecimentos adequados de boas práticas de manipulação (NATARO \& KAPER, 1998). De acordo com OLIVEIRA et al. (2003), a E. coli está entre os principais responsáveis por surtos de toxinfecção alimentar quando associados à condições higiênico-sanitárias insatisfatórias dos manipuladores, como falha na higienização das mãos, indicando contaminação de origem fecal. Portanto, diante dos resultados encontrados neste estudo, a presença destas bactérias nas mãos e na nasofaringe dos manipuladores representa grande importância epidemiológica devido à possibilidade de transferência essas bactérias aos alimentos durante toda a sua cadeia de produção.

Leite cru - Das 24 amostras de leite cru coletadas, 19 (79,2\%) apresentaram contaminação por E. coli, isolando-se 33 cepas. Dados semelhantes foram encontrados em pesquisa realizada por REA et al. (1992) sobre a ocorrência de bactérias indicadoras e patogênicas no leite cru na Irlanda, onde evidenciaram coliformes em $100 \%$ das amostras analisadas, sendo 60\% delas contaminadas por $\boldsymbol{E}$. coli (Tabela 1).

No Estado de São Paulo, BADINI (1993) observou que, de 60 amostras de leite cru, $68,3 \%$ apresentaram contagens de microrganismos mesófilos; destes, $83,3 \%$ foram de coliformes totais e 18,3\% de coliformes fecais. CHYE et al. (2004), pesquisando 360 fazendas de gado leiteiro na Malásia, analisaram 930 amostras de leite cru e relataram que $90 \%$ das amostras continham coliformes e $65 \%$ estavam contaminadas por E. coli com contagens acima dos padrões permitidos.

Segundo LIRA et al. (2004), a carne bovina e o leite cru têm sido confirmados como as fontes mais prováveis de doenças transmitidas por alimentos em surtos investigados na última década, principalmente no Canadá, nos Estados Unidos, no Reino Unido e no Japão. A carga microbiana inicial do leite está diretamente relacionada à higiene da ordenha e à limpeza dos utensílios utilizados para sua coleta e transporte.

Tabela 1 - Contaminação por $\boldsymbol{E}$. coli e amostras obtidas de narizes e mãos de manipuladores, de leite cru e de queijo Minas Frescal coletadas em laticínio de Goiás, Brasil, 2004/2005.

\begin{tabular}{|c|c|c|c|c|c|c|c|c|c|}
\hline \multirow{2}{*}{\multicolumn{2}{|c|}{ Fonte }} & \multirow{2}{*}{\multicolumn{2}{|c|}{$\begin{array}{l}\text { Número de amostras } \\
\text { coletadas }\end{array}$}} & \multicolumn{4}{|c|}{ Amostras positivas } & \multirow{2}{*}{\multicolumn{2}{|c|}{$\begin{array}{c}\text { Número de cepas } \\
\text { isoladas }\end{array}$}} \\
\hline & & & & \multicolumn{2}{|c|}{ Número } & \multicolumn{2}{|c|}{ Freqüência (\%) } & & \\
\hline \multirow{2}{*}{ Manipulador } & Nariz & \multirow{2}{*}{92} & 46 & \multirow{2}{*}{11} & 03 & \multirow{2}{*}{12,0} & 6,5 & \multirow{2}{*}{11} & 03 \\
\hline & Mãos & & 46 & & 08 & & 17,4 & & 08 \\
\hline \multicolumn{2}{|c|}{ Leite cru } & \multicolumn{2}{|c|}{24} & \multicolumn{2}{|c|}{19} & \multicolumn{2}{|c|}{79,2} & \multicolumn{2}{|c|}{33} \\
\hline \multicolumn{2}{|c|}{ Queijo } & \multicolumn{2}{|c|}{24} & \multicolumn{2}{|c|}{17} & \multicolumn{2}{|c|}{70,8} & \multicolumn{2}{|c|}{25} \\
\hline \multicolumn{2}{|c|}{ TOTAL } & \multicolumn{2}{|c|}{140} & \multicolumn{2}{|c|}{47} & \multicolumn{2}{|c|}{-} & \multicolumn{2}{|c|}{69} \\
\hline
\end{tabular}

Ciência Rural, v.36, n.4, jul-ago, 2006. 
Considerando que, neste estudo, 79,2\% das amostras avaliadas apresentaram $\boldsymbol{E}$. coli e que o leite não sofreu nenhum processo de pasteurização para a fabricação do queijo, cepas patogênicas provenientes de contaminação fecal do animal e/ou do manipulador durante a obtenção do leite e a produção do queijo podem ser disseminadas para o consumidor.

Queijo Minas Frescal - Observou-se a presença de $\boldsymbol{E}$. coli em 17 (70,8\%) das 24 amostras de queijo Minas Frescal coletadas, sendo isoladas 25 cepas (Tabela 1).

Resultados semelhantes foram apresentados por PEREIRA et al. (1999), na cidade de Belo Horizonte, os quais observaram $74,3 \%$ de contaminação por coliformes fecais em amostras de queijo Minas Frescal. MONGE et al. (1994) estudaram 20 amostras de queijos comercializados em San José, Costa Rica, identificando $\boldsymbol{E}$. coli em 100\% das amostras e em contagens superiores a $1.100 / \mathrm{g}$.

O elevado percentual de amostras de queijo Minas Frescal apresentando $\boldsymbol{E}$. coli é bastante preocupante, haja vista o risco potencial de causar enfermidades e ainda a possibilidade da presença de outros enteropatógenos como a Salmonella. Os resultados encontrados demonstram que as condições higiênico-sanitárias do produto testado não são satisfatórias, podendo apresentar risco à saúde dos consumidores em razão de sua larga comercialização no Estado.

Diante deste quadro, seria recomendada a atuação mais incisiva dos órgãos de fiscalização sanitária, no intuito de aplicar o que é preconizado na produção deste tipo de queijo, como a pasteurização do leite. É uma prática simples que, se corretamente aplicada, permite a diminuição da carga microbiana inicial com conseqüente eliminação de patógenos. Além disso, a implementação das boas práticas de fabricação minimizaria o perigo da provável contaminação humana e ambiental.

Tipagem por meio de suscetibilidade a antimicrobianos - A suscetibilidade das 69 cepas de $\boldsymbol{E}$. coli isoladas frente aos antimicrobianos testados está demonstrada na tabela 2. Quarenta e dois dos isolados $(60,9 \%)$ foram suscetíveis a todos os antibióticos testados. Destaca-se que, entre os manipuladores, os isolados a partir do nariz apresentaram resistência de $33,3 \%$ à cefalotina, ao sulfametoxazol trimetropim e à tetraciclina. Nenhuma cepa isolada do nariz apresentou resistência à ampicilina, à ciprofloxacina e à gentamicina. As cepas isoladas das mãos apresentaram resistência de $12,5 \%$ para cefalotina e tetraciclina e não foi observada resistência à ampicilina, à ciprofloxacina, à gentamicina e aosulfametoxazol trimetropim.

Em relação ao leite cru, maior resistência $(18,2 \%)$ foi encontrada para tetraciclina, sendo que nenhum isolado apresentou resistência à ciprofloxacina e à gentamicina. Quanto ao queijo Minas Frescal, observou-se resistência $(4,0 \%)$ à ampicilina, ao sulfametoxazol trimetoprim e à tetraciclina e susceptibilidade aos demais antibióticos.

A partir do teste de suscetibilidade para as 69 cepas isoladas, foi possível agrupá-las em 17

Tabela 2 - Suscetibilidade aos antimicrobianos das cepas de $\boldsymbol{E}$. coli isoladas de amostras obtidas de manipuladores, leite cru e de queijo Minas Frescal coletadas em laticínio de Goiás, Brasil, 2004 / 2005.

\begin{tabular}{|c|c|c|c|c|c|c|c|c|}
\hline \multirow{2}{*}{ Fonte } & & \multirow{2}{*}{ Suscetibilidade } & \multicolumn{6}{|c|}{ Antimicrobianos $\mathrm{n}^{\circ}(\%)$} \\
\hline & & & AMP & $\mathrm{CEF}$ & CIP & GEN & SUT & TET \\
\hline \multirow{7}{*}{ 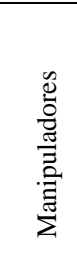 } & \multirow{3}{*}{ Nariz (3) } & $\mathrm{S}$ & $3(100)$ & $1(33,3)$ & $2(66,7)$ & $3(100)$ & $2(66,7)$ & $2(66,7)$ \\
\hline & & SI & $0(0)$ & $1(33,3)$ & $1(33,3)$ & $0(0)$ & $0(0)$ & $0(0)$ \\
\hline & & $\mathrm{R}$ & $0(0)$ & $1(33,3)$ & $0(0)$ & $0(0)$ & $1(33,3)$ & $1(33,3)$ \\
\hline & \multirow{4}{*}{ Mãos (8) } & $\mathrm{S}$ & $8(100)$ & $6(75)$ & $7(87,5)$ & $8(100)$ & $8(100)$ & $6(75)$ \\
\hline & & SI & $0(0)$ & $1(12,5)$ & $1(12,5)$ & $0(0)$ & $0(0)$ & $1(12,5)$ \\
\hline & & $\mathrm{R}$ & $0(0)$ & $1(12,5)$ & $0(0)$ & $0(0)$ & $0(0)$ & $1(12,5)$ \\
\hline & & $\mathrm{S}$ & $29(87,8)$ & $22(66,7)$ & 32 (97) & $33(100)$ & 32 (97) & $23(69,7)$ \\
\hline \multirow{2}{*}{\multicolumn{2}{|c|}{ Leite cru (33) }} & SI & $2(6,1)$ & $7(21,2)$ & $1(3)$ & $0(0)$ & $0(0)$ & $4(12,1)$ \\
\hline & & $\mathrm{R}$ & $2(6,1)$ & $4(12,1)$ & $0(0)$ & $0(0)$ & $1(3)$ & $6(18,2)$ \\
\hline \multirow{3}{*}{\multicolumn{2}{|c|}{ Queijo (25) }} & $\mathrm{S}$ & $24(96)$ & $22(88)$ & $24(96)$ & $24(96)$ & $24(96)$ & $23(92)$ \\
\hline & & SI & $0(0)$ & $3(12)$ & $1(4)$ & $1(4)$ & $0(0)$ & $1(4)$ \\
\hline & & $\mathrm{R}$ & $1(4)$ & $0(0)$ & $0(0)$ & $0(0)$ & $1(4)$ & $1(4)$ \\
\hline
\end{tabular}

* S - sensibilidade; SI - sensibilidade intermediária; R - resistência AMP - ampicilina; CEF - cefalotina; CIP - ciprofloxacina; GEN - gentamicina; SUT - sulfametoxazol-trimetoprin, TET - tetraciclina 
Tabela 3 - Perfis de suscetibilidade a antimicrobianos das cepas de $\boldsymbol{E}$. coli isoladas de amostras de nariz e de mãos de manipuladores, de leite cru e de queijo Minas Frescal coletadas em laticínio de Goiás, Brasil, 2004 / 2005.

\begin{tabular}{|c|c|c|c|c|c|c|c|}
\hline \multirow{2}{*}{ Cepas isoladas } & \multicolumn{6}{|c|}{ Perfis de suscetibilidade a antimicrobianos* } & \multirow{2}{*}{$\begin{array}{c}\text { Perfil } \\
\text { Fenotípico }\end{array}$} \\
\hline & SUT & CIP & AMP & GEN & TET & CFL & \\
\hline 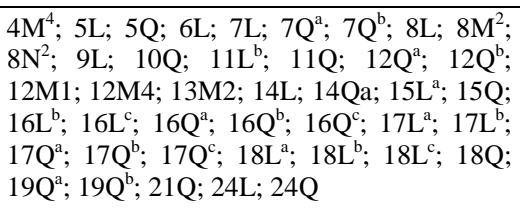 & $\mathrm{S}$ & $\mathrm{S}$ & S & $\mathrm{S}$ & $\mathrm{S}$ & $\mathrm{S}$ & A \\
\hline $5 \mathrm{~L}^{\mathrm{a}} ; 9 \mathrm{Q} ; 11 \mathrm{~L}^{\mathrm{a}} ; 15 \mathrm{M}^{1} ; 16 \mathrm{~L}^{\mathrm{a}} ; 19 \mathrm{~L}^{\mathrm{b}}$ & $\mathrm{S}$ & $\mathrm{S}$ & S & $\mathrm{S}$ & $\mathrm{S}$ & I & B \\
\hline $10 \mathrm{~L}^{\mathrm{b}} ; 13 \mathrm{~L}^{\mathrm{b}} ; 15 \mathrm{~L}^{\mathrm{c}}$ & $\mathrm{S}$ & $\mathrm{S}$ & $\mathrm{S}$ & $\mathrm{S}$ & I & $\mathrm{S}$ & $\mathrm{C}$ \\
\hline $2 \mathrm{~L} ; 20 \mathrm{~N}^{4}$ & $\mathrm{~S}$ & $\mathrm{~S}$ & S & $\mathrm{S}$ & $\mathrm{S}$ & $\mathrm{R}$ & $\mathrm{D}$ \\
\hline $10 \mathrm{M}^{1} ; 14 \mathrm{Q}^{\mathrm{b}}$ & $\mathrm{S}$ & $\mathrm{I}$ & S & $\mathrm{S}$ & $\mathrm{S}$ & $\mathrm{S}$ & $\mathrm{E}$ \\
\hline $11 \mathrm{~L} ; 23 \mathrm{~L}^{\mathrm{b}}$ & $\mathrm{S}$ & S & S & $\mathrm{S}$ & $\mathrm{R}$ & I & $\mathrm{F}$ \\
\hline $15 \mathrm{~L}^{\mathrm{b}} ; 23 \mathrm{~L}^{\mathrm{a}}$ & $\mathrm{S}$ & $\mathrm{S}$ & $\mathrm{S}$ & $\mathrm{S}$ & $\mathrm{R}$ & S & G \\
\hline $3 Q$ & $\mathrm{R}$ & $\mathrm{S}$ & $\mathrm{R}$ & $\mathrm{S}$ & $\mathrm{R}$ & I & $\mathrm{H}$ \\
\hline $4 \mathrm{~L}$ & $\mathrm{~S}$ & $\mathrm{~S}$ & I & $\mathrm{S}$ & $\mathrm{S}$ & $\mathrm{R}$ & I \\
\hline $6 \mathrm{Q}$ & $\mathrm{S}$ & $\mathrm{S}$ & S & I & $\mathrm{S}$ & $\mathrm{S}$ & $\mathrm{J}$ \\
\hline $8 Q^{b}$ & $\mathrm{~S}$ & $\mathrm{~S}$ & S & $\mathrm{S}$ & I & I & $\mathrm{K}$ \\
\hline $8 \mathrm{M}^{1}$ & $\mathrm{~S}$ & $\mathrm{~S}$ & $\mathrm{~S}$ & $\mathrm{~S}$ & I & $\mathrm{R}$ & $\mathrm{L}$ \\
\hline $10 \mathrm{~L}^{\mathrm{a}}$ & $\mathrm{S}$ & I & $\mathrm{S}$ & $\mathrm{S}$ & S & $\mathrm{S}$ & M \\
\hline $10 N^{2}$ & $\mathrm{R}$ & $\mathrm{I}$ & $\mathrm{S}$ & $\mathrm{S}$ & $\mathrm{R}$ & I & $\mathrm{N}$ \\
\hline $12 \mathrm{~L}$ & $\mathrm{~S}$ & $\mathrm{~S}$ & I & $\mathrm{S}$ & $\mathrm{R}$ & I & $\mathrm{O}$ \\
\hline $13 \mathrm{~L}^{\mathrm{a}}$ & $\mathrm{R}$ & $\mathrm{S}$ & $\mathrm{R}$ & $\mathrm{S}$ & $\mathrm{R}$ & $\mathrm{R}$ & $\mathrm{P}$ \\
\hline $19 \mathrm{~L}^{\mathrm{a}}$ & $\mathrm{S}$ & $\mathrm{S}$ & $\mathrm{R}$ & $\mathrm{S}$ & I & $\mathrm{R}$ & $\mathrm{Q}$ \\
\hline
\end{tabular}

* O perfil de suscetibilidade foi baseado na resistência (R), na sensibilidade intermediária (I) e na sensibilidade (S) a antimicrobianos. AMP - ampicilina, CEF - cefalotina, CIP - ciprofloxacina, GEN - gentamicina, SUT - sulfametoxazol-trimetoprin, TET - tetraciclina $\mathrm{L}$ - leite $\quad \mathrm{Q}$ - queijo $\mathrm{M}$ - mão $\left({ }^{1,2,3,4}\right) \quad \mathrm{N}$ - nariz $\left({ }^{1,2,3,4}\right)$.

a, b, c - correspondem a diferentes cepas isoladas de uma mesma amostra de leite cru ou de queijo.

diferentes perfis fenotípicos $(A-Q)$, conforme apresentado na tabela 3. Das cepas isoladas, 42 se apresentaram no perfil A, seis no perfil B, três no perfil $\mathrm{C}$, duas nos perfis $\mathrm{D}$ a $\mathrm{G}$, e os demais perfis com uma cepa cada.

No presente estudo, foi possível identificar apenas um perfil fenotípico (E) semelhante entre cepas de $\boldsymbol{E}$. coli isoladas, tanto de amostra de queijo $\left(14 \mathrm{Q}^{\mathrm{b}}\right)$ como de amostra de um dos manipuladores $\left(10 \mathrm{M}^{1}\right)$. Tal resultado sugere que possa ter ocorrido contaminação do queijo durante a sua manipulação; portanto, apenas neste caso pôde-se estabelecer a origem da $\boldsymbol{E}$. coli para o produto final. Nas demais situações, não foi possível determinar a fonte da bactéria para o queijo, pois, dentro dos mesmos perfis, houve a presença de cepas isoladas tanto do manipulador quanto do leite.

Nas últimas duas décadas, muitos métodos têm sido aplicados para comparar cepas de $\boldsymbol{E}$. coli a fim de identificar os mecanismos de transmissão e fontes de contaminação (ZADOKS et al., 2002). Entre as técnicas fenotípicas, o teste de suscetibilidade a antimicrobianos tem sido especialmente usado devido a seu baixo custo, à facilidade de execução, além de contribuir para informar sobre a resistência microbiana (KLUYTMANS et al., 1995; ACCO et al., 2003).

Porém, os métodos de tipagem fenotípica, que detectam características expressadas pelos microrganismos, são limitados pela capacidade destes em alterar a expressão de genes. Mutações pontuais podem resultar em regulação anormal ou em alteração funcional do gene responsável por um fenótipo determinado. Assim, isolados da mesma espécie e geneticamente indistinguíveis podem apresentar fenótipo variado. Portanto, os testes de resistência a antimicrobianos podem ser úteis como triagem inicial para identificação de cepas de microrganismos em surtos de doenças transmitidas por alimentos, podendo ser complementados com métodos de tipagem genotípica (ARBEIT, 1999; TENOVER et al., 1997). Entretanto, o antibiograma apresenta limitações, como baixa sensibilidade, o que pode levar à determinação de pontos de controle inadequados ou à identificação equivocada de fontes de contaminação.

Ciência Rural, v.36, n.4, jul-ago, 2006. 


\section{CONCLUSÃO}

Considerando os resultados obtidos neste estudo, observou-se que o antibiograma não se mostrou um teste eficiente na determinação da fonte de microrganismos para o produto final, encontrandose apenas um perfil fenotípico que permitiu estabelecer essa relação de contaminação. Apesar desta observação, o antibiograma é ainda eficiente em determinar a suscetibilidade das cepas encontradas nestas fontes e nestes produtos, como também pode ser utilizado como instrumento na triagem para aplicação de testes mais sensíveis, como os de tipagem genotípica.

\section{APROVAÇÃOPORCOMITÊDEÉTICA}

Este artigo é produto de um projeto de pesquisa aprovado pelo Comitê de Ética em Pesquisa da Universidade Federal de Goiás - COEP/UFG.

\section{REFERÊNCIAS}

ACCO, M. et al. Identification of multiple strains of Staphylococcus aureus colonizing nasal mucosa of food handlers. Food Microbiology, v.20, n.5, p.489-493, 2003.

ALMEIDA FILHO, E.S. et al. Perfil microbiológico do queijo tipo Minas Frescal de produção artesanal e inspecionado, comercializado no município de Cuiabá, MT. Higiene Alimentar, São Paulo, v.16, n.92/93, p.51-56, 2002.

ALMEIDA FILHO, E.S.; NADER FILHO, A. Ocorrência de coliformes fecais e Escherichia coli em queijo tipo Minas Frescal de produção artesanal, comercializado em Poços de Caldas, MG. Higiene Alimentar, São Paulo, v.16, n.102/103, p.7173, 2002.

AMMON, A. Surveillance of enterohaemorrhagic $\boldsymbol{E}$. coli (EHEC) infections and haemolytic uraemic syndrome (HUS) in Europe. Eurosurveillance, v.2, n.12, p.91-95, 1997.

ARAÚJO, V.S. et al. Occurrence of Staphylococcus and enteropathogens in soft cheese commercialized in the city of Rio de Janeiro, Brazil. Journal of Applied Microbiology, v.92, n.6, p.1172-1177, 2002.

ARBEIT, R.D. Laboratory procedures for the epidemiologic analysis of microorganisms. In: MURRAY, P.R. et al. Manual of clinical microbiology. Washington: ASM, 1999. Cap.7, p.116-137.

BABÁK, V. et al. Interpretation of the results of antimicrobial susceptibility analysis of $\boldsymbol{E}$. coli isolates from bovine milk, meat and associated foodstuffs. Food Microbiology, v.22, n.4, p.353-358, 2005.

BADINI, K.B. Estudo das características físico-químicas, microbiológicas e dos hábitos de consumo do leite cru comercializado clandestinamente nos municípios de Botucatu -SP e São Manuel - SP. 1993. 111f. Dissertação (Mestrado em Medicina Veterinária) - Faculdade de Ciências Agrárias e Veterinárias, Universidade Estadual Paulista.

BARROS, P.O.G. et al. Avaliação da qualidade microbiológica do queijo Minas Frescal comercializado no município do Rio de Janeiro, RJ. Higiene Alimentar, São Paulo, v.18, n.122, p.57-66, 2004.

BEAN, N.H. et al. Surveillance for foodborne disease outbreaks - United States, 1988 - 1992. Journal of Food Protection, v.60, n.10, p.1265-1286, 1997.

BONFOH, B. et al. Microbiological quality of cow's milk taken at different intervals from the udder to selling point in Bamako (Mali). Food Control, v.14, n.7, p.495-500, 2003.

BRENER, D.J. Facultatively anaerobic Gram-negative rods. In: KRIEG, N.R.; HOLT, J.G. Bergey's manual of systematic bacteriology. Baltimore: Willians \& Wilkins, 1984. Cap.7, p.408-423.

CÂMARA, S.A.V. et al. Avaliação microbiológica de queijos tipo Minas Frescal artesanal, comercializados no mercado municipal de Campo Grande, Mato Grosso do Sul, 2000. Higiene Alimentar, São Paulo, v.16, n.101, p.32-36, 2002.

CARDOSO, L.; ARAÚJO, W.M.C. Parâmetros de qualidade em queijos comercializados no Distrito Federal, no período de 19972001. Higiene Alimentar, São Paulo, v.18, n.123, p.49-53, 2004.

CARDOSO, R.C.V. Avaliação do grau de higiene de manipuladores de alimentos em restaurantes de pólo petroquímico de Camaçari, Salvador. 1999. 138f. Monografia (Especialização em controle de qualidade de alimentos) - Universidade Federal da Bahia.

CHYE, F.Y. et al. Bacteriological quality and safety of raw milk in Malaysia. Food Microbiology, v.21, n.5, p.535-541, 2004.

COWDEN, J.M. et al. Outbreaks of foodborne infectious intestinal disease in England and Wales: 1992 and 1993. Communicable Disease Report, v.5, n.8, p.109-117, 1995.

CURTIS, M.L. et al. Determinación de la calidad microbiológica de alimentos servidos en comedores de empresas privadas. Archivos Latinoamericanos de Nutrición, v.50, n.2, p.177182, 2000.

DE BUYSER, M.L. et al. Implication of milk and milk products in food-borne diseases in France and in different industrialized countries. International Journal of Food Microbiology, v.67, n.1-2, p.1-17, 2001.

DOYLE, M.P. et al. E. coli O157:H7. In: DOYLE, M.P. et al. (Eds). Food microbiology - Fundamentals and frontiers. Washington, DC: ASM, 1997. p.171-191.

FDA - Food \& Drug Administration, 2002. Bacteriological Analytical Manual. Enumeration of Escherichia coli and the Coliform Bacteria. Capturado em 11 ago.2004. Online. Disponível na Internet http//:www.cfsan.fda.gov/ ebam/bam4html. 
FREITAS, A.C. et al. Occurrence and characterization of Aeromonas species in pasteurized milk and white cheese in Rio de Janeiro, Brasil. Journal of Food Protection, v.56, n.5, p.62-65, 1993.

GONZALEZ, A.G.M. et al. Enteropathogenicity markers in Escherichia coli strains isolated from soft white cheese and poultry in Rio de Janeiro, Brazil. Food Microbiology, v.17, n.3, p.321-328, 2000.

IBGE - Pesquisa Trimestral do Leite - Resultados Mensais./ 2003. Quantidade de leite cru ou refriado adquirido Brasil. Capturado em 21 nov. 2005. Online. Disponível na Internet http.//sidra.ibge.gov.br./bda/tabela/ protabl.aso $\mathrm{z}=\mathrm{t} \& \mathrm{o}=3 \& \mathrm{j}=\mathrm{P}$.

KLUYTMANS, J. et al. Food-initiated outbreak of methicillinresistant Staphylococcus aureus analyzed by pheno and genotyping. Journal of Clinical Microbiology, v.33, n.5, p.1121-1128, 1995.

KOTTWITZ, L.B.M.; GUIMARÃES, I.M. Avaliação microbiológica de queijos Coloniais produzidos no Estado do Paraná. Higiene Alimentar, São Paulo, v.17, n.114/115, p.77-80, 2003.

LIRA, W.M. et al. The incidence of shiga toxin-producing Escherichia coli in cattle with mastitis in Brazil. Journal of Applied Microbiology, v.97, n.4, p.861-866, 2004.

LOGUERCIO, A.P.; ALEIXO, J.A.G. Microbiologia de queijo Minas Frescal produzido artesanalmente. Ciência Rural, Santa Maria, v.31, n.6, p.1063-1067, 2001.

MIDURA, T. F.; BRYANT, R.G. Sampling plans, sample collection, shipment, and preparation for analysis. In: VANDERZANT, C.; SPLITTSTOESSER, DF. Compendium of methods for the microbiological examination for foods. Washington: APHA, 2001. p.13-23.

MONGE, R. et al. Incidence of Listeria monocytogenes in pasteurized ice cream and soft cheese in Costa Rica, 1992. Revista de Biologia Tropical, San José, v.42, n.1/2, p.327328, 1994.

NATARO, J.P.; KAPER, J.B. Diarrheagenic Escherichia coli. Clinical Microbiological Reviews, v.11, n.1, p.142-201, 1998.
NCCLS - National Committee for Clinical Laboratory Standards. Approved Standard M2-A8. Performance Standards for Antimicrobial Disk Susceptibility Tests. Wayne, PA: National Committee for Clinical Laboratory Standards, 2003. 31p.

OLIVEIRA, A.M. et al. Manipuladores de alimentos: um fator de risco. Higiene Alimentar, São Paulo, v.17, n.114/115, p.12-19, 2003.

PEREIRA, M.L. et al. Enumeração de coliformes fecais e presença de Salmonella sp. em queijo Minas. Arquivo Brasileiro Medicina Veterinária Zootecnia, v.51, n.5, p.427-431, 1999.

REA, M.C. et al. Incidence of pathogenic bacteria in raw milk in Ireland. Journal of Applied Bacteriology, n.73, p.331336, 1992.

ROOS, T.B. et al. Avaliação microbiológica de queijo colonial produzido na cidade de Três Passos, RS. Higiene Alimentar, São Paulo, v.19, n.132, p.94-96, 2005.

SCHOUTEN, J.M. et al. Prevalence estimation and risk factors for Escherichia coli $\mathrm{O} 157$ on Dutch dairy farms. Preventive Veterinary Medicine, v.64, n.1, p.49-61, 2004.

SILVA JR., E.A. Manual de controle higiênico-sanitário em alimentos. São Paulo: Varela, 2002. 479p.

SIMONE, E. et al. Investigations of foodborne diseases by food inspection services in the Netherlands, 1991 to 1994. Journal of Food Protection, v.60, n.5, p.442-446, 1997.

TENOVER, F.C. et al. How to select and interpret molecular strain typing methods for epidemiological studies of bacterial infections: a review for healthcare epidemiologists. Infection Control and Hospital Epidemiology, v.18, n.6, p.426-439, 1997.

ZADOKS, R.N. et al. Comparison of Staphylococcus aureus isolates from bovine and human skin, milking, equipment, and bovine milk by phage typing, pulsed-field gel electrophoresis, and binary typing. Journal of Clinical Microbiology, v.40, n.11, p.3894-3902, 2002. 